\title{
Angiotensin-(1-7) inhibits the migration and invasion of A549 human lung adenocarcinoma cells through inactivation of the PI3K/Akt and MAPK signaling pathways
}

\author{
LEI NI $^{1 *}$, YUN FENG $^{1 *}$, HUANYING WAN $^{1}$, QINYUN MA ${ }^{2}$, LIANG FAN ${ }^{1}$, \\ YANRONG QIAN $^{1}$, QINGYUN LI $^{1}$, YI XIANG $^{1}$ and BEILI GAO ${ }^{1}$ \\ ${ }^{1}$ Department of Respiration, and ${ }^{2}$ Center of Molecular Medicine, Shanghai Institute of \\ Endocrinology, State Key Laboratory of Medical Genomics, Ruijin Hospital, Shanghai Jiaotong \\ University School of Medicine, 197 Ruijin Road II, Shanghai 200025, P.R. China
}

Received September 30, 2011; Accepted October 29, 2011

DOI: 10.3892/or.2011.1554

\begin{abstract}
The local renin-angiotensin system (RAS) is one of the crucial components in the tumor microenvironment. Recent evidence suggests that the local RAS plays an important role in tumor metabolism, survival, angiogenesis and invasion processes. Angiotensin-(1-7) [Ang-(1-7)] is an endogenous peptide of the RAS with vasodilator and anti-proliferative properties. Previous studies have demonstrated that Ang-(1-7) inhibits both the growth of human lung cancer cells in vitro and tumor angiogenesis in vivo through activation of the MAS receptor. This study investigated the anti-metastatic effect of Ang-(1-7) in A549 human lung adenocarcinoma cells in vitro. We found that Ang-(1-7) reduced the cell migratory and invasive abilities by reducing the expression and activity of MMP-2 and MMP-9. Furthermore, we demonstrated that the anti-migration and anti-invasion effect of Ang-(1-7) was mediated through inactivation of the PI3K/Akt, P38 and JNK signal pathways. Our results suggest that Ang-(1-7) may have therapeutic potential against advanced lung carcinoma as a new agent.
\end{abstract}

\section{Introduction}

Lung cancer is the leading cause of cancer deaths worldwide (1), and non-small cell lung cancer (NSCLC) accounts for almost $80 \%$ of all lung cancers. Despite intense efforts to improve lung cancer treatment, the overall 5-year survival

Correspondence to: Dr Huanying Wan, Department of Respiration, Ruijin Hospital, Shanghai Jiaotong University School of Medicine, 197 Ruijin Road II, Shanghai 200025, P.R. China

E-mail: hywan_fy@yahoo.com.cn

${ }^{*}$ Contributed equally

Key words: angiotensin-(1-7), MMPs, PI3K/Akt, MAPK, non-small cell lung cancer in patients with advanced NSCLC remains approximately $10-15 \%$ (2). This grim prognosis is mainly attributed to its early local advance and distant metastasis. The factors that regulate the metastatic process of lung cancer, however, are poorly understood. Thus, there is a need for new therapeutic targets and a better understanding of the mechanisms involved in the metastasis of lung cancer.

Emerging data suggest that, in addition to systemically produced angiotensin, local RAS system contributes importantly to tumor angiogenesis and tumor progression, and tumor environment contains all RAS components necessary to produce angiotensin locally (3). Recent experimental and clinical evidence indicated that the RAS components are involved in the pathophysiology of tumors $(4,5)$. In our previous studies (6), we demonstrated that angiotensin-converting enzyme2 (ACE2) overexpression can decrease angiotensin II (Ang-II) levels and suppress the proliferation in human lung adenocarcinoma cell lines. Ang-(1-7), mainly generated from Ang-II by the ACE2, is an endogenous 7 aa peptide hormone with vasodilator, antiproliferative and antithrombotic properties (7). These physiologic effects are exerted through activation of a unique $\mathrm{G}$ protein-coupled Ang-(1-7) receptor encoded by the MAS gene (8). Furthermore, Ang-(1-7) was reported to cause a marked decrease in DNA synthesis and cell proliferation of human lung cancer cells in a dose- and time-dependent manner and to inhibit tumor angiogenesis in vivo by reducing vascular endothelial growth factor-A $(9,10)$. These studies suggested that Ang-(1-7), a component of RAS system, maybe a novel therapeutic agent for lung cancer treatment. However, previous studies of Ang-(1-7) have been mainly focused on the effect of inhibiting tumor cell proliferation or angiogenesis, the role of Ang-(1-7) in metastasis has not been addressed.

The formation of distant metastasis is the main cause of mortality in patients with cancer. Tumor metastasis consists of a complex cascade of interdependent steps, such as cellto-cell and cell-to-extracellular matrix (ECM) interactions, degradation of ECM components, vessel formation, local intravasation invasion, dissemination, extravasation and metastasis formation, and is regulated by extremely complicated mechanisms (11). MMPs are a group of zinc-dependent 
proteases that are produced by tumor cells or the surrounding stroma cells and are involved in the degrading and remodeling of ECM (12). Among them, matrix metalloproteinase-2 (MMP-2) and matrix metalloproteinase-9 (MMP-9) (also known as gelatinase A and B) efficiently degrade IV collagen, the major component of basement membrane, have been suggested to be critical factors for the invasive and metastatic potential in lung cancer (13). Elevated expression of MMP-2 and MMP-9 has been detected in lung cancer tissues and cancer cells in culture (14).

Numerous reports have demonstrated that MMP expression is mediated by mammalian mitogen-activated protein kinases (MAPK) members and the phosphatidylinositol 3-kinase (PI3K)/Akt pathway $(15,16)$. MAPK superfamily, activated by growth factors, cytokines, and cell-matrix interactions, convert signals from triggered cell membrane receptors into a wide range of cellular responses, and play an important regulatory role in cell growth, apoptosis, differentiation and metastasis. Extracellular signal regulated kinase 1 and 2 (ERK1/2), c-Jun N-terminal kinase 1 and 2 (JNK1/2), and p38 MAPK, these three major mammalian MAP kinases play a central role in regulating the expression of MMPs $(17,18)$. In addition, PI3K/ Akt signal transduction pathway regulates cell metastasis and is closely associated with the development and progress of various tumors. The activation of PI3K/Akt and its downstream factors, have been reported to increase the expression of MMPs and promote tumor invasion $(19,20)$. Since these signaling pathways mediate metastasis through the regulation of MMPs expression, the regulatory pathways of MMPs have been considered as promising targets for anti-metastasis therapy agents.

Based on these observations, our study was performed to investigate the inhibitory effects of Ang-(1-7) on the migration and invasion of human lung adenocarcinoma A549 cells in vitro, and to examine the related signaling pathways that are targeted in this context.

\section{Materials and methods}

Materials and chemicals. Ang-(1-7) and specific Ang-(1-7) receptor antagonist [D-Ala $]$-Ang-(1-7) (A-779) were purchased from Bachem. Ham's F-12 was purchased from Invitrogen Life Technologies. Matrigel was purchased from BD Biosciences. MMP-2, MMP-9, p38, JNK, ERK1/2, and $\beta$-actin polyclonal antibodies were purchased from Santa Cruz Biotechnology (Santa Cruz, CA, USA). Antibodies against PI3K, phosphoPI3K, Akt, phospho-Akt, phospho-p38, phospho-JNK, and phosphor-ERK1/2 were from Cell Signaling Technology (Beverly, MA, USA). A549 lung cancer cells were obtained from Shanghai Institute of Cells.

Cell culture. A549 human lung adenocarcinoma cells, were grown in Ham's F-12 medium with $10 \%$ fetal bovine serum, $100 \mu \mathrm{g} / \mathrm{ml}$ penicillin and 100 units $/ \mathrm{ml}$ streptomycin. Cell preparation was carried out at $37^{\circ} \mathrm{C}$ with a humidified atmosphere of $5 \% \mathrm{CO}_{2}$. Culture medium was changed every 3 days, and the treatment of Ang-(1-7) and A-779 was renewed each day. The cells were plated onto 6-well culture plates for the measurement of the mRNA and protein expression.
Wound-healing assay. For cell migration determination, A549 cells, treated with Ang-(1-7) at various concentrations (25, $50,100 \mathrm{nM})$ or with Ang-(1-7) (100 nM) in the presence of A-779 (100 nM), or with PBS as control, were grown to $80 \%$ confluence in 6-well dishes for $48 \mathrm{~h}$ (three dishes per group). The cells in monolayer were disrupted by scraping them with a P100 micropipette tip, after scraping, the cells were washed twice with PBS ( $\mathrm{pH} 7.4)$. At the indicated times (0-48 h), the number of cells in the denuded zone of each dish was counted and photographed at x100 magnification (Olympus IX71, Nikon DS-U1 camera, Japan) in a blinded fashion. Migrated cells across the white lines were counted in six random fields from each triplicate treatment, and the data are presented as mean \pm SD.

In vitro cell invasion assay. The ability of A549 cells on invasion to pass through Matrigel $^{\mathrm{TM}}$ Matrix-coated filters was measured by the Boyden chamber invasion assay (Corning, NY, USA). Polycarbonate filters (pore size, $8 \mu \mathrm{m}$ ) were precoated with Matrigel, which was diluted to $200 \mu \mathrm{g} / \mathrm{ml}$ with cold filtered distilled water and applied to the top side of the polycarbonate filter. In brief, A549 cells were incubated with Ang-(1-7) at various concentrations $(25,50,100 \mathrm{nM})$ or with Ang-(1-7) (100 nM) in the presence of A-779 (100 nM), or with PBS as control for $48 \mathrm{~h}$. Subsequently, the cells were detached by trypsin, and were seeded onto the upper chamber in $200 \mu \mathrm{l}$ serum-free medium containing different treatment. Medium containing $10 \%$ FBS-medium was applied to the lower chamber as chemoattractant, and then the chamber were incubated in a humidified atmosphere with $5 \% \mathrm{CO}_{2}$ at $37^{\circ} \mathrm{C}$ for $48 \mathrm{~h}$. At the end of incubation, cells that invaded across the Matrigel and migrated to the lower surface of the filter were fixed and stained with $2 \%$ crystal violet in $2 \%$ ethanol. The cells on the upper side of the filter were carefully removed by wiping with a cotton swab. The invasive cells on the underside of the filter were counted in randomly selected fields and recorded with an Olympus BX50 inverted microscope and Nikon DS-Ri1 camera at x100 magnification. The data are presented as the average number of cells attached to the bottom surface from randomly chosen fields. Each experiment was repeated three times.

Gelatin zymography. The activities of MMP-2 and MMP-9 in the conditioned medium were measured by gelatin-zymogram protease assays. Briefly, the prepared samples $(20 \mu \mathrm{g}$ total protein) from each treatment group were mixed with loading buffer and electrophoresed on $8 \%$ SDS-polyacrylamide gel containing $0.1 \%$ gelatin. After electrophoresis, gels were washed twice with zymography washing buffer (2.5\% Triton $\mathrm{X}-100,50 \mathrm{mM}$ Tris- $\mathrm{HCl}, 5 \mathrm{mM} \mathrm{CaCl}{ }_{2}, \mathrm{pH}$ 7.6) on a gyratory shaker for $30 \mathrm{~min}$ at room temperature to remove SDS, followed by incubation at $37^{\circ} \mathrm{C}$ for $12 \mathrm{~h}$ in zymography reaction buffer (40 mM Tris-HCl, pH 8.0, $10 \mathrm{mM} \mathrm{CaCl}_{2}, 0.02 \% \mathrm{NaN}_{3}$ ). The gels were stained with Coomassie blue R-250 $(0.125 \%$ Coomassie blue R-250, 0.1\% amino black, 50\% methanol, $10 \%$ acetic acid) for $2 \mathrm{~h}$, and destained with destaining solution (20\% methanol, $10 \%$ acetic acid, $70 \%$ double-distilled $\mathrm{H}_{2} \mathrm{O}$ ). Non-staining bands representing the levels of the latent form of MMP-2 and MMP-9 were quantified by densitometer measurement using a digital imaging analysis system. 
Real-time quantitative reverse transcription analysis. Total RNA of treated cells was extracted using TRIzol ${ }^{\mathrm{TM}}$ reagent (Promega Corp.) according to the supplier's instructions. RNA was quantitated by optical density measurement at 260 and $280 \mathrm{~nm}$ using a spectrophotometer (all RNA samples had an A260/A280 ratio $>1.8$ ), and integrity was confirmed by running RNA on a $1.2 \%$ agarose gel. Reverse transcription was performed with $1 \mu \mathrm{g}$ of total RNA using Reverse Transcription System (Takara Shuzo, Shiga, Japan). Real-time PCR was performed using SYBR-Green Supermix with an iCycler ${ }^{\circledR}$ thermal cycler (Bio-Rad). Primers were obtained from Shanghai Sangon Biological Engineering Technology and Services (Shanghai, China) and their sequences were: MMP-2 forward: 5'-CCCTGATGTCCAGCG-3', reverse: 5'-AGCAGCCTAGCCAGTC-3'; MMP-9 forward: 5'-CAC TGTCCACCCCTCAGAGC-3', reverse: 5'-GCCACTTGT CGGCGATAAGG-3'; P38 forward: 5'-GGTCTGTTGGA CGTTTTTAC-3', reverse: 5'-TAGGTTTTAGGTCCCTG TGA-3'; JNK forward: 5'-TGAGAAACTCTTCCCTGATG-3', reverse: 5'-GCTTCAGAAGGATCATACCA-3'; ERK forward: 5'-GGAAGCATTATCTTGACCAG-3', reverse: 5'-CTCT TGTGTGCGTTGAATGT-3'; PI3K forward: 5'-AGGTT CATGTGCTGGATACT-3', reverse: 5'-TGGGCTCCTT TACTAATCTC-3'; Akt forward: 5'-ACGATGAATGAGGT GTCTGT-3', reverse: 5'-TCTGCTACGGTGAAGTTGTT-3'; GAPDH forward: 5'-GGTCGGAGTCAACGGATTTG-3', reverse: 5'-ATGAGCCCCAGCCTTCTCCAT-3'. The threshold cycle $(\mathrm{Ct})$ for each PCR product was calculated with the instrument's software, and obtained $\mathrm{Ct}$ values were normalized by subtracting the $\mathrm{Ct}$ values of GAPDH. The resulting $\Delta \mathrm{Ct}$ values were then used to calculate the relative change in mRNA expression as a ratio $(\mathrm{R})$ according to the $2^{-\Delta \Delta \mathrm{Ct}}$ equation method.

Western blot analysis. Expression of MMP-2, MMP-9, MAPK and PI3K/Akt signaling protein (P38, p-P38, JNK1/2, p-JNK1/2, ERK1/2, p-ERK1/2, PI3K, p-PI3K, Akt, p-Akt, $\beta$-actin) levels in A549 cells was studied by Western blot analysis. A549 cells in F12K medium were placed in culture plates, and then treated with Ang-(1-7) at various concentration (25, $50,100 \mathrm{nM})$, or with Ang-(1-7) (100 nM) in the presence of A-779 (100 nM), or with PBS as control for $48 \mathrm{~h}$. For total cell protein extracts, control and treated cells were washed in PBS, suspended in lysis buffer containing $50 \mathrm{mM}$ Tris- $\mathrm{HCl}(\mathrm{pH} 7.5)$, $1 \% \mathrm{NP}-40,2 \mathrm{mM}$ EDTA, $10 \mathrm{mM} \mathrm{NaCl}, 10 \mu \mathrm{g} / \mathrm{ml}$ aprotinin, $10 \mu \mathrm{g} / \mathrm{ml}$ leupeptin, $1 \mathrm{mM}$ DTT, $0.1 \%$ SDS, $1 \mathrm{mM}$ PMSF and placed in ice for $30 \mathrm{~min}$. After centrifugation for $15 \mathrm{~min}$ at $4^{\circ} \mathrm{C}$, the supernatant was collected. Protein concentrations were determined using the BCA assay (Pierce, Rockford, IL, USA). For Western blot analysis, equal amounts of proteins $(50 \mu \mathrm{g})$ were separated by SDS-polyacrylamide gel electrophoresis and transferred to a nitrocellulose membrane. The blot was blocked in blocking buffer (5\% non-fat dry milk and $1 \%$ Tween-20 in PBS) for $1 \mathrm{~h}$ at room temperature, and then incubated with appropriate primary antibodies in blocking buffer overnight at $4^{\circ} \mathrm{C}$. The blot was then incubated with appropriate secondary antibody alkaline phosphatase (AP) conjugated and detected in $5 \mathrm{ml}$ AP buffer containing $16.5 \mu \mathrm{l}$ BCIP and $33 \mu \mathrm{l} \mathrm{NBT}$ at room temperature for 10-20 min, and then photographed. $\beta$-actin was used as a loading control.
Statistical analysis. Data are presented as means \pm SD. Student's t-test was used to compare the differences between two groups. Differences were considered statistically significant at $\mathrm{P}<0.05$ (two-tailed). All statistical analyses were conducted using SPSS version 11.0 (SPSS Inc., Chicago, IL).

\section{Results}

Effects of Ang-(1-7) on cell migration. To evaluate the antimetastatic activity of Ang-(1-7) in vitro, we first assessed the inhibitory effect of Ang-(1-7) on A549 cell migration by wound-healing assay. As shown in Fig. 1, according to the quantitative assessment, A549 cells exposed to 50 and $100 \mathrm{nM}$ Ang-(1-7) expressed a significantly reduced ability to migrate and fill the wounded area as compared with the control, and the anti-migration effect was blocked by the combination treatment of $100 \mathrm{nM}$ Ang-(1-7) and $100 \mathrm{nM} \mathrm{A-779,} \mathrm{the} \mathrm{MAS}$ receptor antagonist. These results revealed that Ang-(1-7) significantly inhibited the migration ability of A549 cells in a dose-dependent manner.

Effects of Ang-(1-7) on the cell invasion. One important characteristic of metastasis is the migratory and invasive ability of tumor cells. We used Boyden chamber assay to evaluate the effect of Ang-(1-7) on the invasive ability of A549 cells. The results showed that the number of cells invaded to the lower chamber was significantly reduced by treatment of Ang-(1-7) in a dose-dependent manner (Fig. 2). According to the quantitative assessment, A549 cells treated with 50 and $100 \mathrm{nM}$ of Ang-(1-7) expressed a reduced ability of invasion as compared with the control, and the anti-invasive effect of Ang-(1-7) was reversed by the combination of A-779. These results demonstrated that Ang-(1-7) significantly inhibited the invasive ability of A549 cells in a dose-dependent manner.

Effects of Ang-(1-7) on the MMP expressions of A549 cells. Real-time quantitative RT-PCR analysis and Western blotting was performed to determine the effect of Ang-(1-7) on MMP expression. An analysis of qRT-PCR data of MMP-2 and MMP-9 mRNA, corrected for GAPDH as an internal control (Fig. 3A), showed that the expression of MMP-2 mRNA in 50 and $100 \mathrm{nM}$ Ang-(1-7) treated A549 cells was significantly lower than in control cells, however, there was no change in the expression of MMP-9. In the Western blot analysis (Fig. 3B), we found the same result, Ang-(1-7) significantly suppressed the expression of MMP2 protein in a dose-dependent manner, but not for MMP-9, and A-779 blocked the active effect of Ang-(1-7).

Effects of Ang-(1-7) on the MMP activities of A549 cells. Since the activation of MMPs, which is required for the cell migration and invasion processes, is crucial for ECM degradation, the effect of Ang-(1-7) on the gelatinase activity was further investigated. The conditioned medium in each treatment group was collected, concentrated and assayed for MMPs activity by gelatin zymography. As shown in Fig. 4, MMP-2 and MMP-9 activities were greatly reduced by Ang-(1-7) at 50 and $100 \mathrm{nM}$ concentrations, and A-779 blocked the active effect of Ang-(1-7). The results indicated that the enzyme activities 

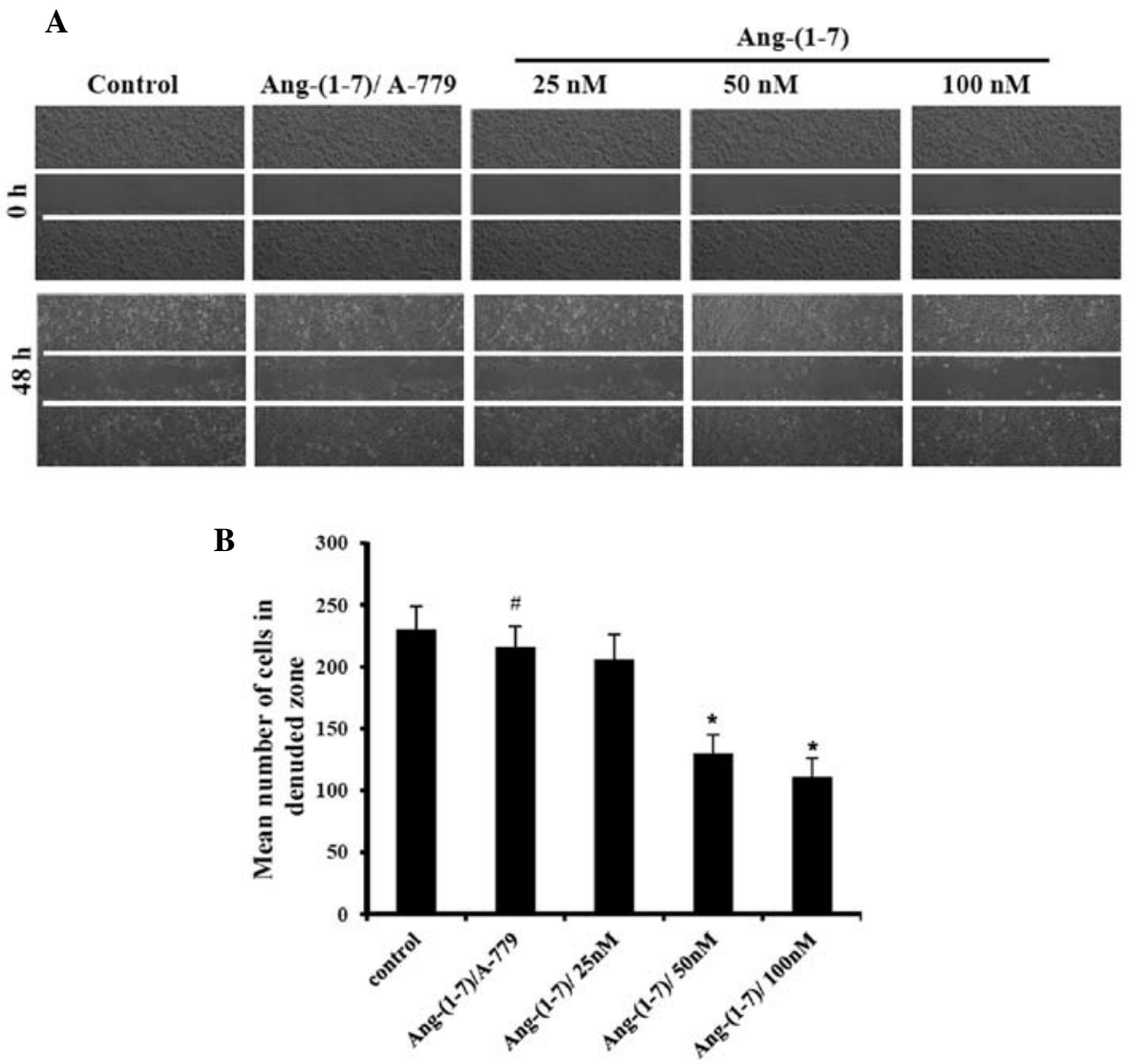

Figure 1. Effects of Ang-(1-7) on A549 cell migration. (A) Monolayers of A549 cells in each treatment group were scraped and the number of cells in the denuded zone was photographed and quantified $48 \mathrm{~h}$ after wound-healing. White lines indicate the wound edge. (B) The mean number of migrated cells across the white lines in the denuded zone was counted in six random fields from each treatment. Values are expressed as mean \pm SD of three independent experiments. ${ }^{*} \mathrm{P}<0.05$ vs. control. ${ }^{\prime \prime} \mathrm{P}<0.05$ vs. $100 \mathrm{nM}$ Ang-(1-7) treatment group.

$\mathbf{A}$

\begin{tabular}{|c|c|c|c|}
\hline \multirow[b]{2}{*}{ Ang-(1-7)/A-779 } & \multicolumn{3}{|c|}{ Ang-(1-7) } \\
\hline & $25 \mathrm{nM}$ & $50 \mathrm{nM}$ & $100 \mathrm{nM}$ \\
\hline
\end{tabular}

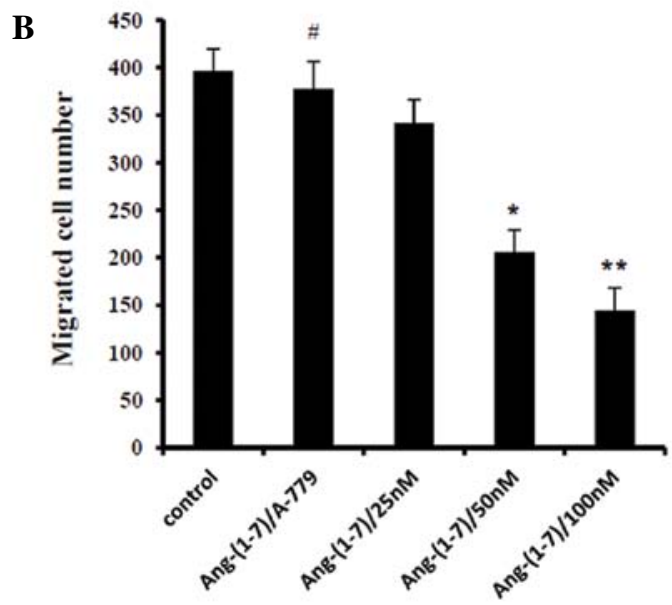

Figure 2. Effects of Ang-(1-7) on A549 cell invasion. (A) In vitro invasion assay was performed by using Boyden chamber coated with Matrigel. Invaded cell number was determined at $48 \mathrm{~h}$ after cell seeding. (B) Migration and invasion ability of A549 cells were quantified by counting the number of the cells that invaded to the underside of the porous polycarbonate membrane. Values are expressed as mean $\pm \mathrm{SD}$ of three independent experiments. ${ }^{*} \mathrm{P}<0.05,{ }^{* *} \mathrm{P}<0.01 \mathrm{vs}$. control. ${ }^{~} \mathrm{P}<0.05$ vs. $100 \mathrm{nM}$ Ang-(1-7) treatment group. 
A

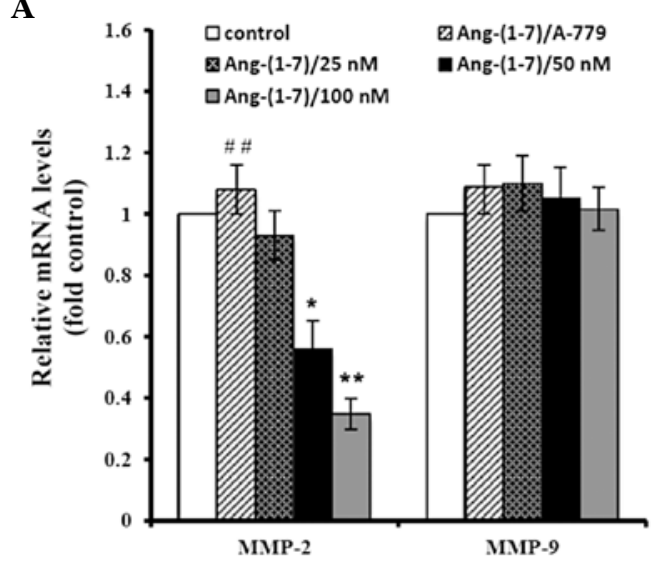

B control Ang-(1-7) Ang-(1-7)

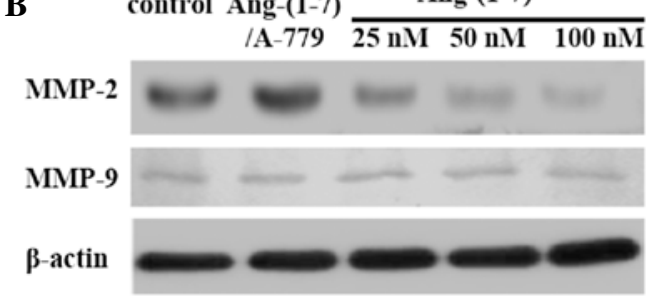

Figure 3. Effects of Ang-(1-7) on MMP expression in A549 cells. (A) Realtime quantitative RT-PCR was performed for MMP-2 and MMP-9, the relative mRNA levels were normalised to GAPDH mRNA expression and then compared to control group. The expression levels of these mRNAs are presented relative to the value of control (mean $\pm \mathrm{SD},{ }^{*} \mathrm{P}<0.05,{ }^{* * *} \mathrm{P}<0.01$ vs. control. ${ }^{\# \#} \mathrm{P}<0.01$ vs. $100 \mathrm{nM}$ Ang-(1-7) treatment group). (B) In Western blot analysis, expression of MMP-2 and MMP-9 protein in cultured A549 cells treated with different concentrations of Ang-(1-7) and with a combination of $100 \mathrm{nM}$ Ang-(1-7) and $100 \mathrm{nM} \mathrm{A-779.} \beta$-actin served as an internal control of protein level.

of MMP-2 and MMP-9 were inhibited by Ang-(1-7) in a dosedependent manner.

Effects of Ang-(1-7) on PI3K/Akt and MAPK signaling pathway in A549 cells. PI3K/Akt and MAPK signaling pathway are involved in the expression of MMPs and inducing metastasis. In order to investigate the anti-metastasis mechanism of Ang-(1-7), the effects of Ang-(1-7) on the expression and phosphorylated status of PI3K/Akt and MAPK signaling in A549 cells were investigated by real-time quantitative RT-PCR and Western blot analysis. The mRNA expression of PI3K/Akt and MAPK signaling was not affected with Ang-(1-7) treatment (Fig. 5A and B). The total protein levels of PI3K/Akt and MAPK signaling were not changed with Ang-(1-7) treatment, while the phosphorylation of PI3K/Akt, JNK1/2 and p38 MAPK signaling were significantly inhibited by Ang-(1-7) as compared with the control (Fig. 5C and D). In addition, these active effects were blocked by the combination with A-779. The data revealed that the inhibition of the expression and activity of MMP-2 and MMP-9 by Ang-(1-7) on A549 cells could partly occur through PI3K/Akt, JNK and p38 MAPK inactivation.

\section{Discussion}

To our knowledge, the present study is the first to demonstrate the anti-metastasis effect of Ang-(1-7) in vitro. We found that


Figure 4. Effects of Ang-(1-7) on the gelatinolytic activity of MMPs in A549 cells. (A) Gelatin zymography assay was performed to analyze the MMP activity, gelatinolytic activity of MMP-2 and MMP-9 was visualized as clear bands against the dark background of stained gelatin. (B) MMP-2 and MMP-9 activities were quantified by densitometric analysis. The densitometric data were expressed as mean $\pm \mathrm{SD}$ of three independent experiments. ${ }^{*} \mathrm{P}<0.05$, ${ }^{* * *} \mathrm{P}<0.01$ vs. control. ${ }^{\#} \mathrm{P}<0.05,{ }^{\# \#} \mathrm{P}<0.01$ vs. $100 \mathrm{nM}$ Ang-(1-7) treatment group.

Ang-(1-7) inhibit human lung adenocarcinoma A549 cell migration and invasion through reduction of the expression and enzymatic activity of MMPs, which was regulated by inactivation of PI3K/Akt and MAPK signaling pathways, and the active effects of Ang-(1-7) were blocked by the special MAS receptor antagonist A-779. Our findings strengthen the potential of Ang-(1-7) and its MAS receptor as a novel strategy for anti-cancer therapy.

Local RAS is one of the components of the tumor microenvironment. Recent advances in the understanding of cancer metastasis suggest that the ability of cancer cells to metastasis is acquired early in tumorigenesis and is regulated to a large extent by the tumor micro-environment (21). There is evidence that angiotensin-converting enzyme (ACE) also participates locally in the pathology of carcinomas. ACE is differentially expressed in several tumor types and Ang-II, generated from angiotensinogen by ACE, influences tumor cell proliferation, migration, angiogenesis, and metastatic behavior $(22,23)$. ACE2 is a newly identified component of RAS, with $42 \%$ amino acid homology to ACE. Our previous study confirmed that ACE2 protein expression was decreased in NSCLC tissues in which Ang-II was higher than the matching non-malignant tissues, and the statistical analysis of ACE2 expression and the clinical features of patients with NSCLC showed that ACE2 expression was closely correlated to clinical stage and smoking status, we further found that overexpression of ACE2 has antitumor effects via inhibition of angiogenesis and tumor cell invasion in vivo and in vitro $(5,24)$. ACE2 converts Ang-II to Ang-(1-7), which, contrary to Ang-II, has 
A

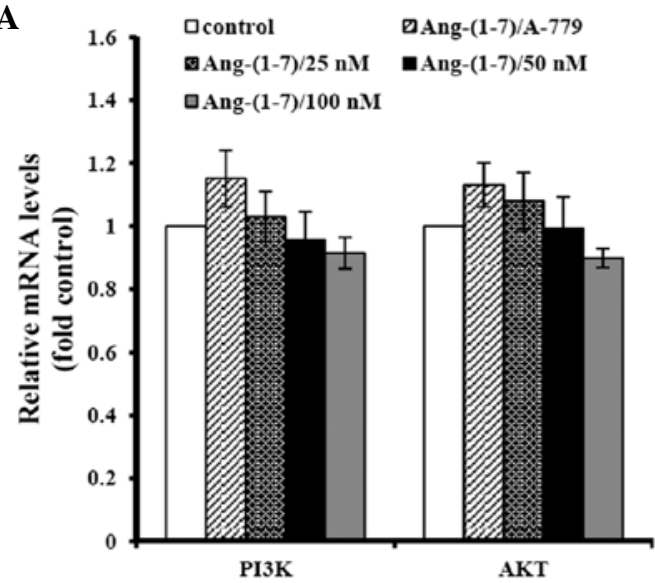

C

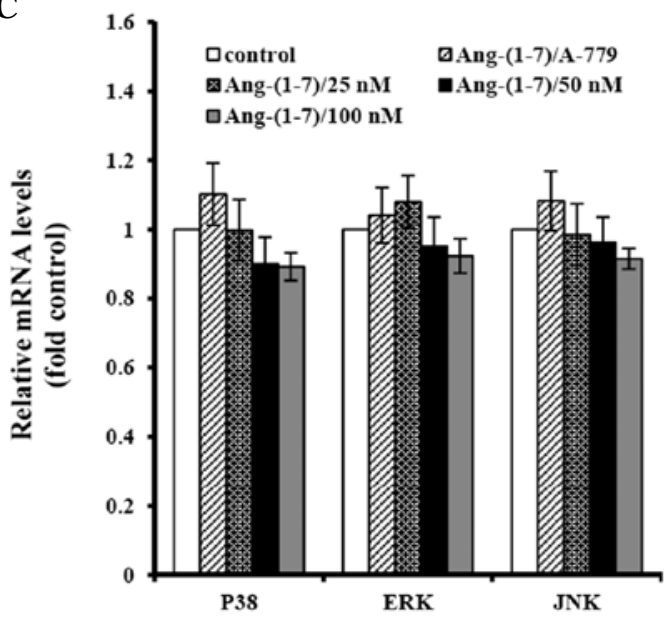

B

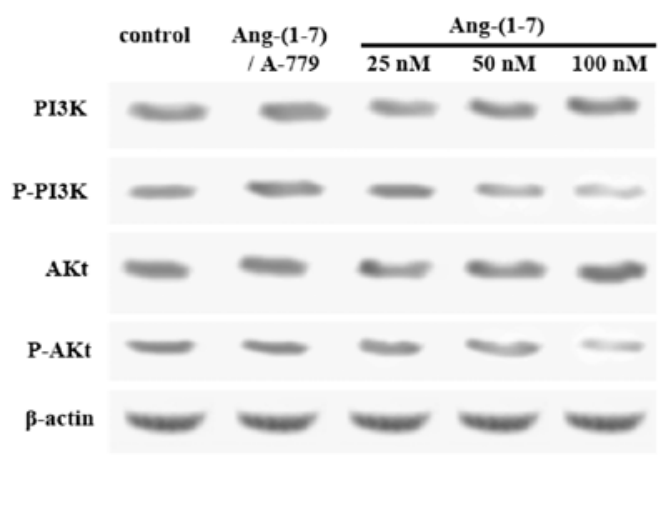

D

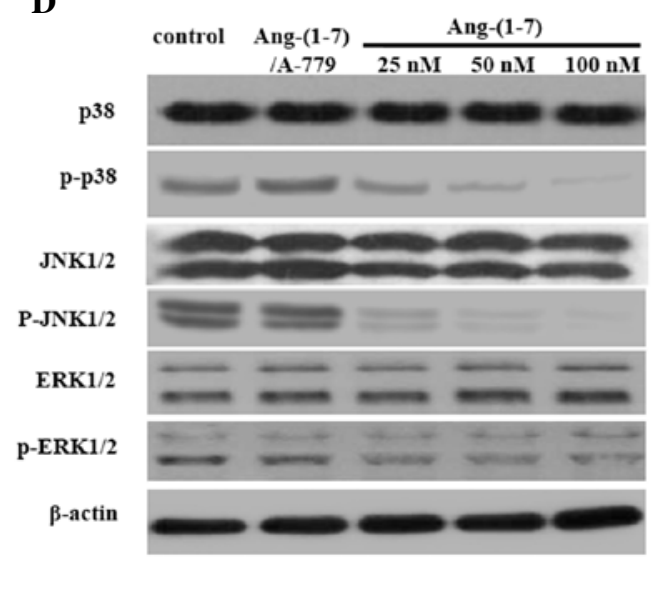

Figure 5. Effects of Ang-(1-7) on PI3K/Akt and MAPK signaling in A549 cells. (A) Quantitative real-time PCR was performed for PI3K/Akt signaling, NAPDH served as an internal control. Values are expressed as mean \pm SD of three independent experiments. (B) The protein levels of PI3K/Akt (PI3K, phospho-PI3K, Akt, phospho-Akt) were determined by Western blot analysis. $\beta$-actin served as an internal control. (C) Quantitative real-time PCR was performed for MAPK signaling (p38, JNK and ERK). (D) The protein levels of MAPK signaling (p38, phospho-p38, JNK1/2, phospho-JNK1/2, ERK1/2, phospho-ERK1/2) were determined by Western blot analysis. $\beta$-actin served as an internal control.

an antiproliferative and anti-angiogenic activity. As proposed, the local RAS can be envisioned as a dual function system, which is primarily driven by the ACE/ACE2 balance (3). Based on these studies, we first explored effect of Ang-(1-7) as a regulator of cell migration and invasion, and as a potential pathway regulator.

Cancer cell-matrix interaction is an initial and critical step for promoting cell migration and invasion, excess ECM degradation is one of the hallmarks of tumor invasion and migration (25). MMPs play an important role in ECM degradation for tumor growth, invasion and tumor-induced angiogenesis. In the present study, wound-healing assay and Boyden chamber assay were applied to investigate the migration and invasive ability of A549 cells in vitro. The result demonstrated that treatment with Ang-(1-7) at 50 and $100 \mathrm{nM}$ concentration exerted an inhibitory effect on the migration and invasion of the highly metastatic human lung adenocarcinoma cells. To investigate the possible mechanisms, we performed real-time RT-PCR and Western blot assays to detect the effect of Ang-(1-7) on the expression of MMP-2 and MMP-9. Furthermore, gelatin zymographic assays were used to examined the detection of collagenase activities. Our result demonstrated that Ang-(1-7) inhibited MMP-2 through different mechanisms, which include reduced expression of MMP-2 genes and inhibition of the enzymatic activity. It is also noteworthy that the gelatinase activity of MMP-9 was inhibited, whereas its mRNA and protein level was not affected. Since the activity of MMPs is controlled by naturally endogenous inhibitors the tissue inhibitors of matrix metalloproteinases (TIMPs), TIMP-1 selectively binds MMP-9 and inhibit its activity (26). There is evidence that, as opposed to Ang-II, Ang-(1-7) treatment leads to decrease the ratio of expression of MMPs/TIMPs in human cardiocytes (27). Ang-(1-7) may inhibit A549 cell migration and invasion through a complex regulation of MMPs/TIMPs, which is worth further investigation.

MMP expression is critically mediated by MAPK and PI3K/Akt pathway (15). Identifying the key proteins involved in these processes is vital for understanding carcinogenesis and to devise new therapies. A recent study showed that the metastasis process of NSCLC cells is regulated by PI3K/Akt signaling pathway (28). In agreement with these reports, we observed that Ang-(1-7) caused a dose-dependent decrease in cellular level of phosphorylated PI3K and Akt. In addition, our result demonstrated that Ang-(1-7) inhibited 
phosphorylation of JNK and P38 signaling, which indicated a potential mechanism of the inhibition of MMP synthesis. JNK and p38 MAPK pathways, also called stress activated protein kinase pathways, are activated by environmental stress and have important roles in inflammation, as well as in tissue homeostasis (29,30). There is evidence that Ang-(1-7) may inhibit lung tumor growth through decreasing COX-2 activity, and COX-2 is considered as a procarcinogenic agent which attribute to inflammation, angiogenesis, and tumor growth (31). Combined with these data, we suggest that Ang-(1-7), a peptide with anti-inflammatory properties, plays an important role in the regulation of tumor micro-environment, and the inactivation of PI3K/Akt and MAPK signaling pathway might be responsible for the inhibitory effect of Ang-(1-7).

Two different ways contribute to the Ang-(1-7): inhibition of the activity of ACE or upgrade of the expression of ACE2. A number of epidemiological evidence suggests protective effects of ACE inhibitors (ACEI) on certain types of cancer (32-34). Another clinical observational study showed that ACEI leads to a longer overall survival in patients with advanced NSCLC undergoing chemotherapy (35). ACEI, currently in widespread use for the treatment of hypertension, causes a reduction in Ang-II and a significant elevation in both tissue and circulating Ang-(1-7) (36). Based on this evidence, the reduced cancer risk observed in patients after administration of ACEI may be due, at least in part, to the elevated levels of Ang-(1-7). Ang-(1-7) exerts its antiproliferative and anti-invasive effects through activation of the special MAS receptor, and A-779, a selective antagonist for MAS receptor, blocks most responses to Ang-(1-7) (37). Our results suggest that the regulators of RAS such as ACEI, ACE2, MAS receptor may provide a clue for the development of novel therapeutic strategies to advanced lung cancer. Further studies using animal models may be helpful to investigate the anti-metastasis effect of Ang-(1-7) in lung cancer.

In summary, we have demonstrated for the first time that Ang-(1-7) inhibited A549 cell migration and invasion through inactivation of JNK, P38 and PI3K/Akt signaling pathway. It reduced the expression of MMP-2 and decreased the enzyme activity of both MMP-2 and MMP-9. Given our previous studies that ACE2 is down-regulated in lung cancer, we suggest that local RAS is an important regulator in lung cancer micro-environment, ACE2-Ang-(1-7)-MAS receptor axis might be a promising target for anti-cancer drugs and chemopreventive agents.

\section{Acknowledgements}

This study was financially supported by the grant from National Natural Science Foundation of China (81071925).

\section{References}

1. Jemal A, Bray F, Center MM, Ferlay J, Ward E and Forman D: Global cancer statistics. CA Cancer J Clin 61: 69-90, 2011.

2. Erridge SC, Moller H, Price A and Brewster D: International comparisons of survival from lung cancer: pitfalls and warnings. Nat Clin Pract Oncol 4: 570-577, 2007.

3. Santos RA, Ferreira AJ and Simões e Silva AC: Recent advances in the angiotensin-converting enzyme 2-angiotensin-(1-7)-Mas axis. Exp Physiol 93: 519-527, 2008.

4. Deshayes F and Nahmias C: Angiotensin receptors: a new role in cancer? Trends Endocrinol Metab 16: 293-299, 2005.
5. Lau ST and Leung PS: Role of the RAS in pancreatic cancer. Curr Cancer Drug Targets 11: 412-420, 2011.

6. Feng Y, Wan H, Liu J, Zhang R, Ma Q, Han B, Xiang Y, Che J, Cao H, Fei X and Qiu W: The angiotensin-converting enzyme 2 in tumor growth and tumor-associated angiogenesis in non-small cell lung cancer. Oncol Rep 23: 941-948, 2010.

7. Ferrario CM, Trask AJ and Jessup JA: Advances in biochemical and functional roles of angiotensin-converting enzyme 2 and angiotensin-(1-7) in regulation of cardiovascular function. Am J Physiol Heart Circ Physiol 289: H2281-H2290, 2005.

8. Santos RA, Simoes e Silva AC, Maric C, et al: Angiotensin-(1-7) is an endogenous ligand for the G protein-coupled receptor Mas. Proc Natl Acad Sci USA 100: 8258-8263, 2003.

9. Gallagher PE and Tallant EA: Inhibition of human lung cancer cell growth by angiotensin-(1-7). Carcinogenesis 25: 2045-2052, 2004.

10. Soto-Pantoja DR, Menon J, Gallagher PE and Tallant EA: Angiotensin-(1-7) inhibits tumor angiogenesis in human lung cancer xenografts with a reduction in vascular endothelial growth factor. Mol Cancer Ther 8: 1676-1683, 2009.

11. Fidler IJ: The organ microenvironment and cancer metastasis. Differentiation 70: 498-505, 2002.

12. Egeblad M and Werb Z: New functions for the matrix metalloproteinases in cancer progression. Nat Rev Cancer 2: 161-174, 2002.

13. Parks WC and Shapiro SD: Matrix metalloproteinases in lung biology. Respir Res 2: 10-19, 2001

14. Thomas P and Khokha R: Diferential expression of matrix metalloproteinases and their inhibitors in non-small cell lung cancer. J Pathol 190: 150-156, 2000.

15. Chakraborti S, Mandal M, Das S, Mandal A and Chakraborti T: Regulation of matrix metalloproteinases: an overview. Mol Cell Biochem 253: 269-285, 2003.

16. Reddy KB, Nabha SM and Atanaskova N: Role of MAP kinase in tumor progression and invasion. Cancer Metastasis Rev 22: 395-403, 2003.

17. Lee SO, Jeong YJ, Im HG, Kim CH, Chang YC and Lee IS: Silibinin suppresses PMA-induced MMP-9 expression by blocking the AP-1 activation via MAPK signaling pathways in MCF-7 human breast carcinoma cells. Biochem Biophys Res Commun 354: 165-171, 2007.

18. Lopez-Bergami P, Huang C, Goydos JS, Yip D, Bar-Eli M, Herlyn M, Smalley KS, Mahale A, Eroshkin A, Aaronson S and Ronai Z: Rewired ERK-JNK signaling pathways in melanoma. Cancer Cell 11: 447-460, 2007.

19. Vivanco I and Sawyers CL: The phosphatidylinositol 3-kinase AKT pathway in human cancer. Nat Rev Cancer 2: 489-501, 2002.

20. Kim D, Kim S, Koh H, Yoon SO, Chung AS, Cho KS and Chung J: $\mathrm{Akt} / \mathrm{PKB}$ promotes cancer cell invasion via increased motility and metalloproteinase production. FASEB J 15: 1953-1962, 2001.

21. Sahai E: Illuminating the metastatic process. Nat Rev Cancer 7: 737-749, 2007.

22. Juillerat-Jeanneret L, Celerier J, Chapuis Bernasconi C, et al: Renin and angiotensinogen expression and functions in growth and apoptosis of human glioblastoma. Br J Cancer 90: 1059-1068, 2004.

23. Zhou L, Zhang RF, Zhang LF, Yao WY, Li JS and Yuan YZ: Angiotensin-converting enzyme 2 acts as a potential molecular target for pancreatic cancer therapy. Cancer Lett 307: 18-25, 2011.

24. Feng Y, Ni L, Wan H, et al: Overexpression of ACE2 has antitumor effects via inhibition of angiogenesis and tumor cell invasion in vivo and in vitro. Oncol Rep 26: 1157-1164, 2011.

25. Noel A, Jost M and Maquoi E: Matrix metalloproteinases at cancer tumor - host interface. Semin Cell Dev Biol 19: 52-60, 2008.

26. Brew K, Dinakarpandian D and Nagase H: Tissue inhibitors of metalloproteinases: evolution, structure and function. Biochim Biophys Acta 1477: 267-283, 2000.

27. Pan $\mathrm{CH}$, Wen $\mathrm{CH}$ and Lin CS: Interplay of angiotensin II and angiotensin-(1-7) in the regulation of matrix metalloproteinases of human cardiocytes. Exp Physiol 93: 599-612, 2009.

28. Chien CS, Shen KH, Huang JS, Ko SC and Shih YW: Antimetastatic potential of fisetin involves inactivation of the $\mathrm{PI} 3 \mathrm{~K} / \mathrm{Akt}$ and JNK signaling pathways with downregulation of MMP-2/9 expressions in prostate cancer PC-3 cells. Mol Cell Biochem 333: 169-180, 2009.

29. Nebreda AR and Porras A: p38 MAP kinases: beyond the stress response. Trends Biochem Sci 25: 257-260, 2000. 
30. Kyriakis JM and Avruch J: Mammalian mitogen activated protein kinase signal transduction pathways activated by stress and inflammation. Physiol Rev 81: 807-869, 2001.

31. Menon J, Soto-Pantoja DR, Callahan MF, Cline JM, Ferrario CM, Tallant EA and Gallagher PE: Angiotensin-(1-7) inhibits growth of human lung adenocarcinoma xenografts in nude mice through a reduction in cyclooxygenase-2. Cancer Res 67: 2809-2814, 2007.

32. Lever AF, Hole DJ and Gillis CR: Do inhibitors of angiotensinI-converting enzyme protect against risk of cancer? Lancet 352: 179-184, 1998

33. Christian JB, Lapane KL, Hume AL, Eaton CB and Weinstock MA: Association of ACE inhibitors and angiotensin receptor blockers with keratinocyte cancer prevention in the randomized VATTC trial. J Natl Cancer Inst 100: 1223-1232, 2008.

34. Arafat HA, Gong Qk, Chipitsyna G, Rizvi A, Saa CT and Yeo CJ: Antihypertensives as novel antineoplastics: angiotensinI-converting enzyme inhibitors and angiotensin II type 1 receptor blockers in pancreatic ductal adenocarcinoma. J Am Coll Surg 204: 996-1005, 2007.
35. Wilop S, von Hobe S, Crysandt M, Esser A, Osieka R and Jost E: Impact of angiotensin I converting enzyme inhibitors and angiotensin II type 1 receptor blockers on survival in patients with advanced non-small cell lung cancer undergoing first-line platinum-based chemotherapy. J Cancer Res Clin Oncol 135: 1429-1435, 2009.

36. Luque M, Martin P, Martell N, Fernandez C, Brosnihan KB and Ferrario CM: Effects of captopril related to increased levels of prostacyclin and angiotensin-(1-7) in essential hypertension. J Hypertens 14: 799-805, 1996.

37. Tallant EA, Ferrario CM and Gallagher PE: Angiotensin-(1-7) inhibits growth of cardiac myocytes through activation of the mas receptor. Am J Physiol Heart Circ Physiol 289: 1560-1566, 2007. 